\title{
Optical Design for the Submillimeter and Far InfraRed Experiment (SAFIRE)
}

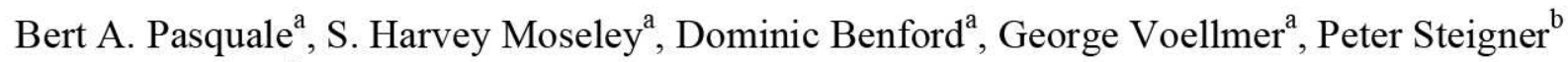 \\ ${ }^{a}$ NASA/Goddard Space Flight Center, Greenbelt, MD 20771 \\ ${ }^{\mathrm{b}}$ Bastion Technologies, Inc., Lanham, MD 20706
}

\begin{abstract}
The SAFIRE, the Submillimeter and Far InfraRed Experiment, was designed for interstellar physics in the airborne Observatory SOFIA. SAFIRE is a cryogenic Echelle Grating spectrograph for covering 27 to 470 microns, with R ranging from 2-6,000. Here we will discuss the details of the optical design, the design process, and the performance of the instrument.
\end{abstract}

Keywords: Optical Design, Instrumentation, Spectroscopy, High-resolution, SOFIA, Far-Infrared

\section{INTRODUCTION}

The SAFIRE concept has undergone many design over the past decade, this latest incarnation described here uses interchangeable dual diffraction gratings to process bands from 27 to 470 microns, though other bands outside these ranges are also possible. While previous concepts for this science have utilized a Fabry-Perot design, [1] this design is diffraction based using a ruled grating, providing a robust and stable system. The emphasis on galaxy astrophysics drives the grating selection, [2] but the design allows for specific wavebands to be targeted for highest resolution for any application. This system was designed specifically for the mechanical and operational constraints of the airborne observatory SOFIA, the Stratospheric Observatory For Infrared Astronomy, shown in Figure 1.

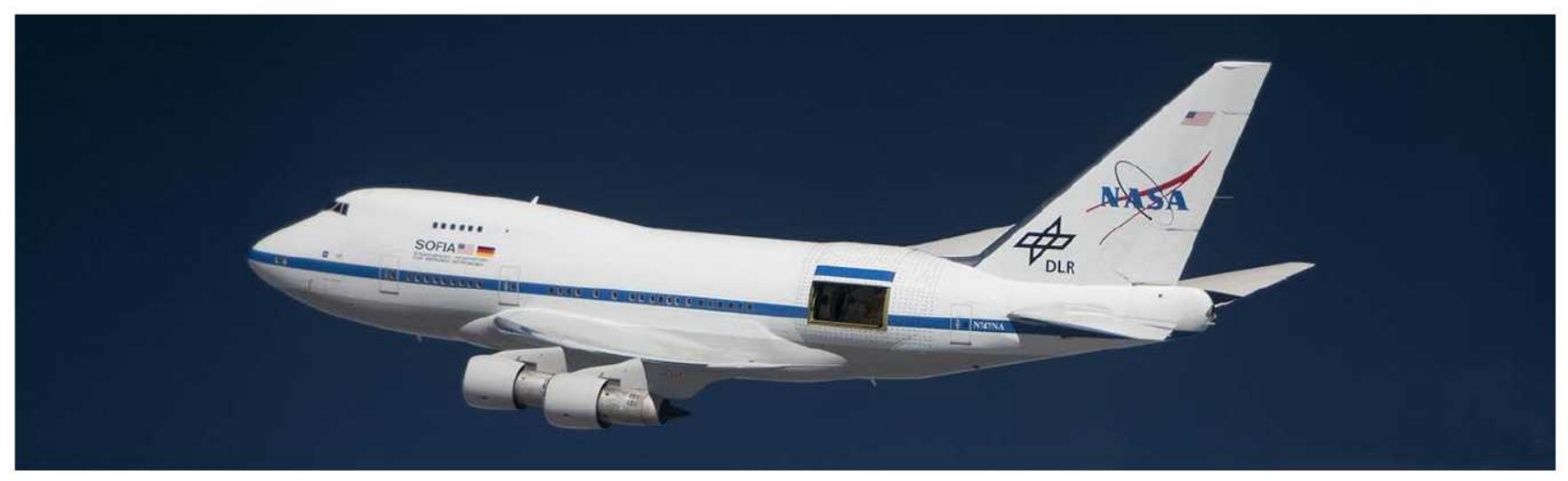

Figure 1. The SOPHIA observatory. (Photo Credit: NASA/Jim Ross)

\section{OPTICAL LAYOUT}

\subsection{SOFIA Observatory}

SOFIA carries a $2.5 \mathrm{~m}$ optical telescope (See Figure 2) that can be coupled with various instruments in the plane's fuselage, as done so with SAFIRE in Figure 3. With tight volume constraints, creating an instrument to operate within the boundaries is a challenge, especially when seeking to do fast, high-resolution spectroscopy. 


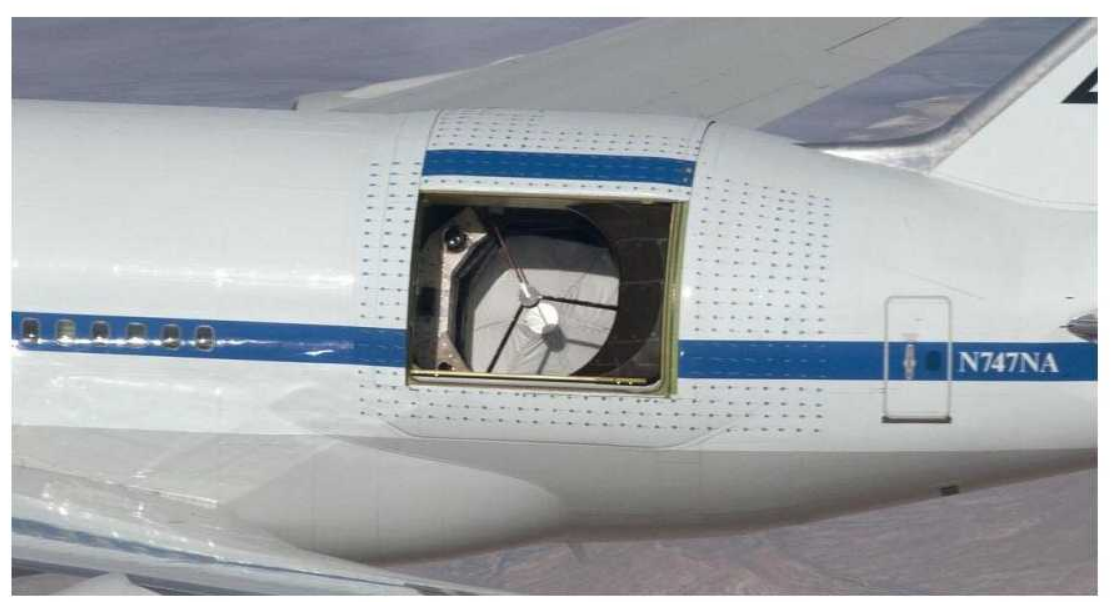

Figure 2. The SOPHIA 100-inch (2.5m) telescope. (Photo Credit: NASA/Carla Thomas)

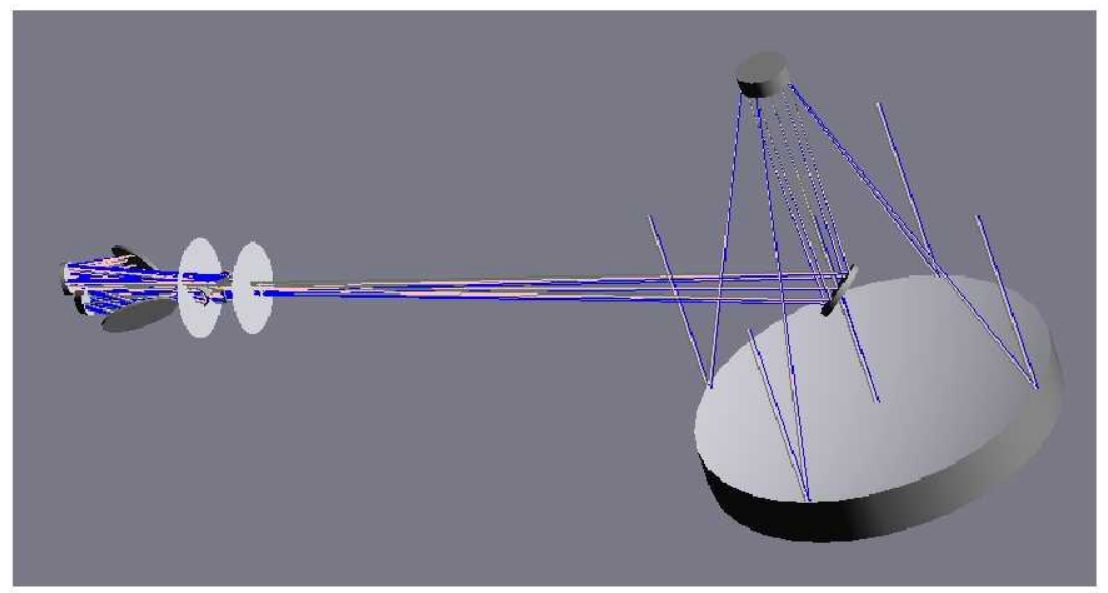

Figure 3. The SOPHIA Telescope \& SAFIRE instrument optical system Integration.

\subsection{SOPHIA Thermal Shroud}

SAFIRE operates within a cryogenically cooled shroud, producing further constraints on the volume (Figure 4.)

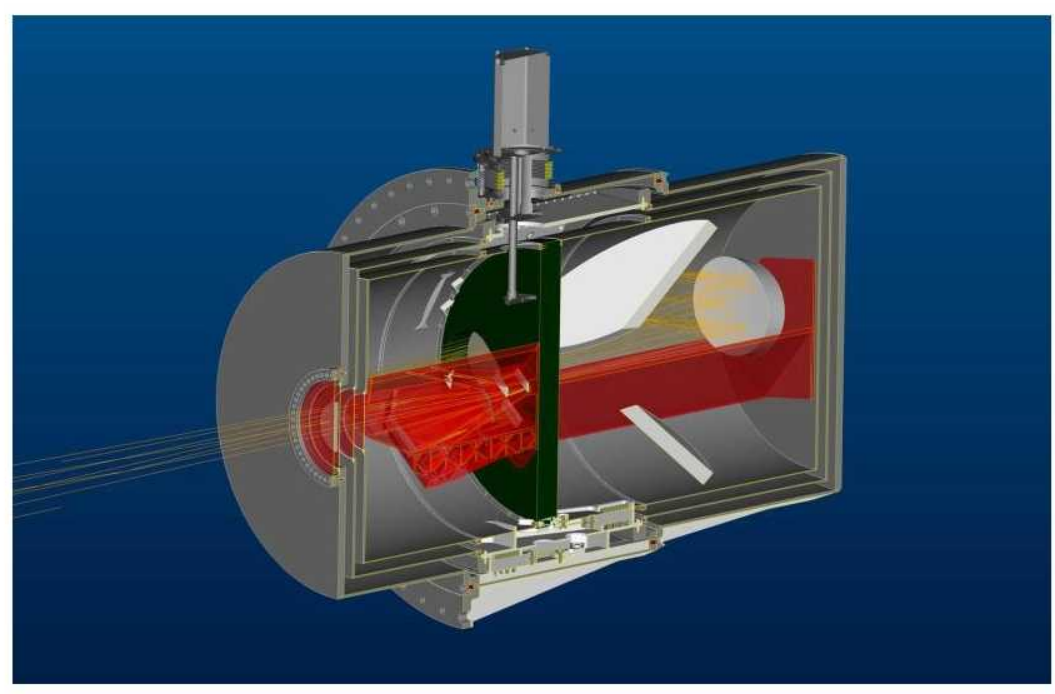

Figure 4. SAFIRE and housing, Within the SOPHIA thermal shroud 


\subsection{Fore Optical System}

Figure 5 shows the details of the optical components. The optical system begins (1) before the image from SOFIA is formed, splitting the image (either via an amplitude beamsplitter or a dichroic) into two optically identical and independent channels (2) and using a slit to limit the extent of light entering each channel (3).

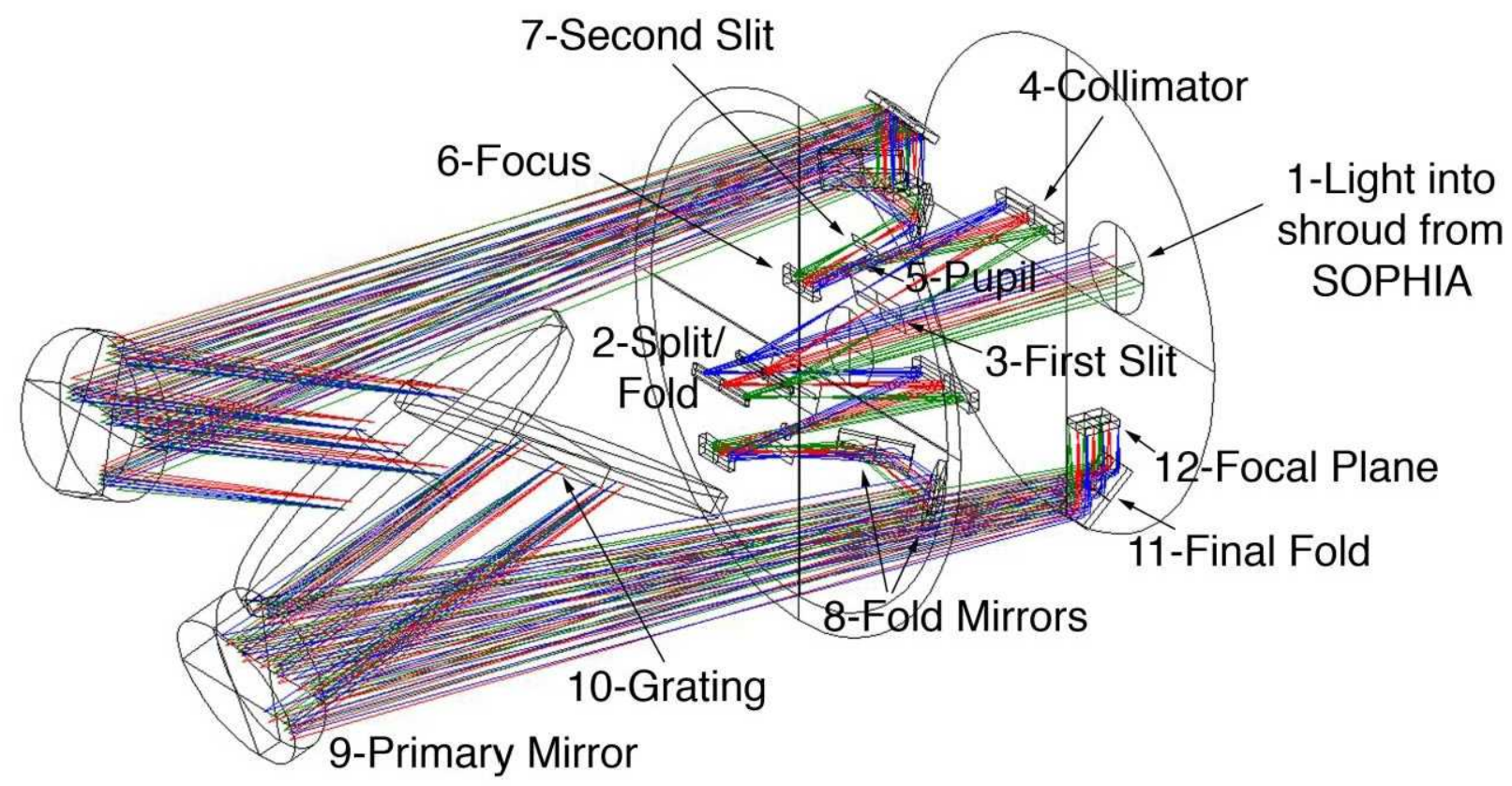

Figure 5. SAFIRE Instrument Optical System.

Figure 6 continues showing the optical beam path. The beams are collimated (4), and passed through a bandpass filter at a pupil (5). A powered mirror (6) then reimages the input field to another slit (7), and also sets the distance to the next pupil. The second slit is the system field stop, and will directly affect the spectral resolution and throughput.

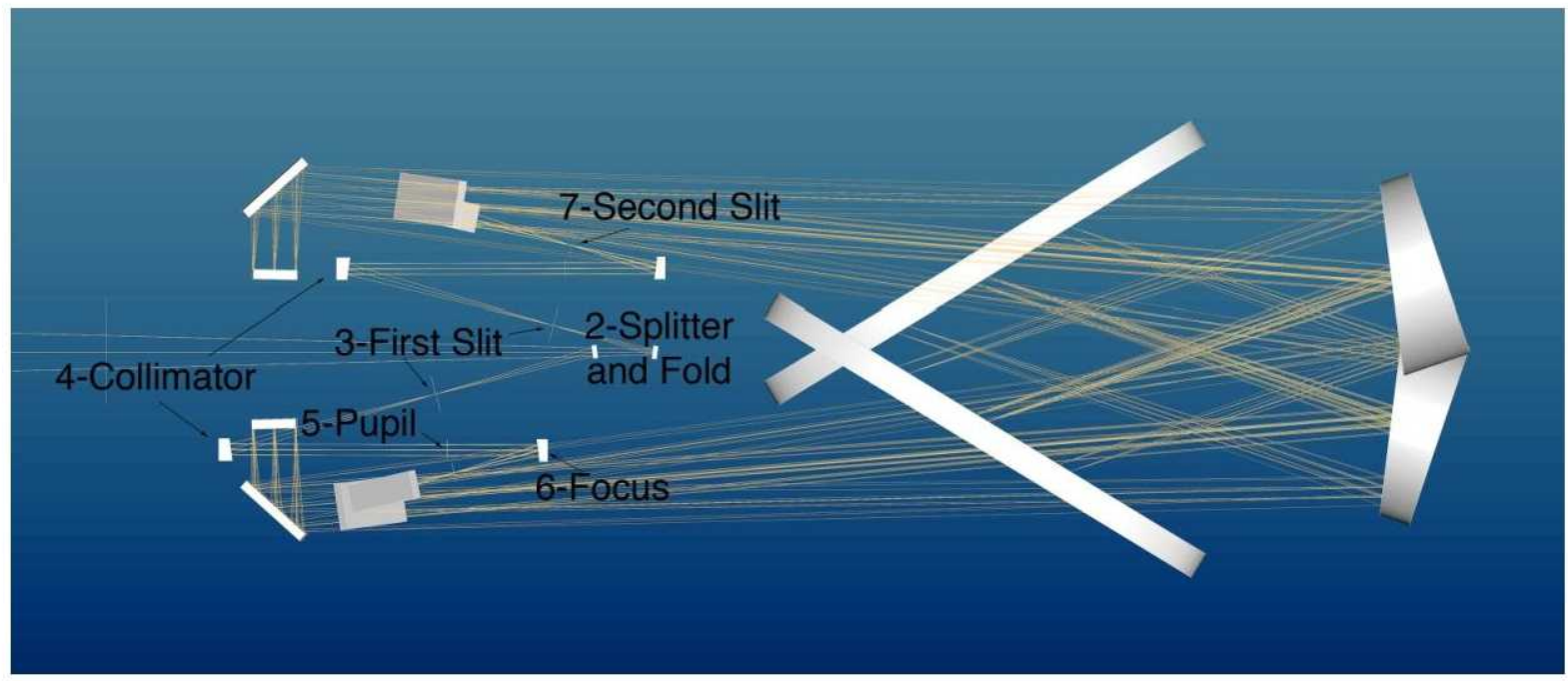

Figure 6. SAFIRE optical system, with gratings in minimum dispersion position ( $40^{\circ}$ incident.) 


\subsection{Spectrometer and Aft Optical System}

After reflecting off two fold mirrors (8), the light then expands to the instrument primary mirror (9), where it is collimated and sent to the grating (10). The grating is located at the next relayed pupil location for minimum physical size.

The grating operates between $40^{\circ}$ and $70^{\circ}$ (Figure $6 \&$ Figure 7 show the gratings at the $40^{\circ}$ incident/diffracted position), and diffracted light is sent back to the primary mirror with a slight angular offset. The final beam is reimaged by the primary mirror (9) off a final fold mirror (11) to the detector (12), with 32 spatial pixels and 40 spectral pixels.

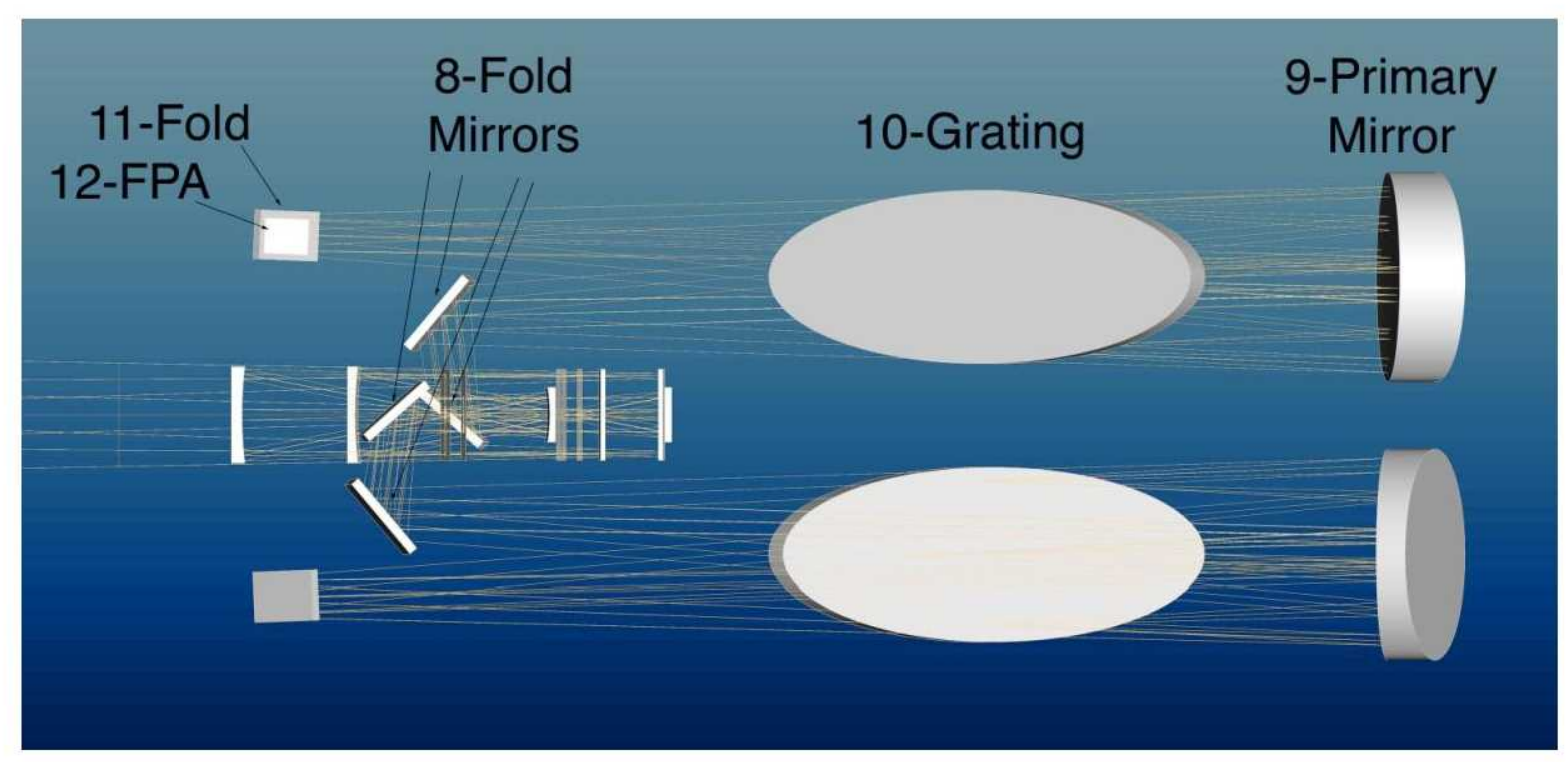

Figure 7. SAFIRE optical system, highlighting the dual grating channels.

\section{OPTICAL DESIGN PROCESS}

The optical system is built off the output from the SOFIA Telescope. This introduces the first design constraint of the location of the telescope focal plane to the available space for the instrument. Because of this, we chose to split and reflect the incoming beam in advance of the telescope image plane, to make use of the aft area of the available space.

Dual channels in the instrument are optically identical, though specific path differences in the two channels may differ between flat (fold) mirrors. All optics described apply to either of the channels.

Following the channel split, the telescope image is first imaged through a slit. This slit may be oversized for the system, and may be further clipped at the second slit. The expanding light is collimated to a beam size of about $11.6 \mathrm{~mm}$, and a pupil is formed $240 \mathrm{~mm}$ away.

The pupil allows a small bandpass filter to be inserted that matches the grating range. The pupil field spread produces an angular range of $\pm 10^{\circ}$ at the pupil.

The focus mirror creates the image for the instrument slit into the spectrometer. The power of the mirror is determined by two principles: First, the beam expansion must allow the ray bundle enough distance to clear the grating, but it must grow enough to fill the desired aperture of the primary mirror. Its conjugate distance was also chosen to relay the pupil an appropriate distance to minimize the grating size. The slit can be adjusted to the desired width, depending on wavelength and desired balance of throughput \& resolution. (The effect of this will be discussed in Section 4.) 
The two fold mirrors are optically neutral elements but do play an important role in the alignment of the spectrometer. First, they are use to balance out the path lengths so the two primary mirrors are re-aligned to the same axial distance and decenter. Second, they also provide rotation so the two spectrometers are "in plane" to the rest of the optical bench.

The primary mirrors are dual-purpose off-axis parabolas with a $90 \mathrm{~mm}$ clear aperture. First, they collimate the beam to the gratings, then they refocus the beam back to the detectors. A slight offset allows the incoming and outgoing beams to pass by each other without requiring a beamsplitter.

The interchangeable gratings are each configured in concert with the desired bandpass and slit configurations, to be operated in $1^{\text {st }}$ order between $40^{\circ}-70^{\circ}$ with equal angle of diffraction. The grating dimension is the same, no matter what the wavelength range is. The grating is elliptical with dimensions $270 \mathrm{~mm} \times 95 \mathrm{~mm}$, though smaller gratings could be used (with reduction in resolution.)

As the grating is rotated about the front face of the minor axis, the wavelength that is returning back to the primary mirror is modulated. The primary mirror now images both the spatial slit width, and a range of spectral height to the image plane. This will be shown in the next section.

\section{PERFORMANCE}

The SAFIRE system provides a consistent image quality across both the spatial and spectral fields, (see Figure 8) and becomes diffraction limited above $80 \mu \mathrm{m}$.
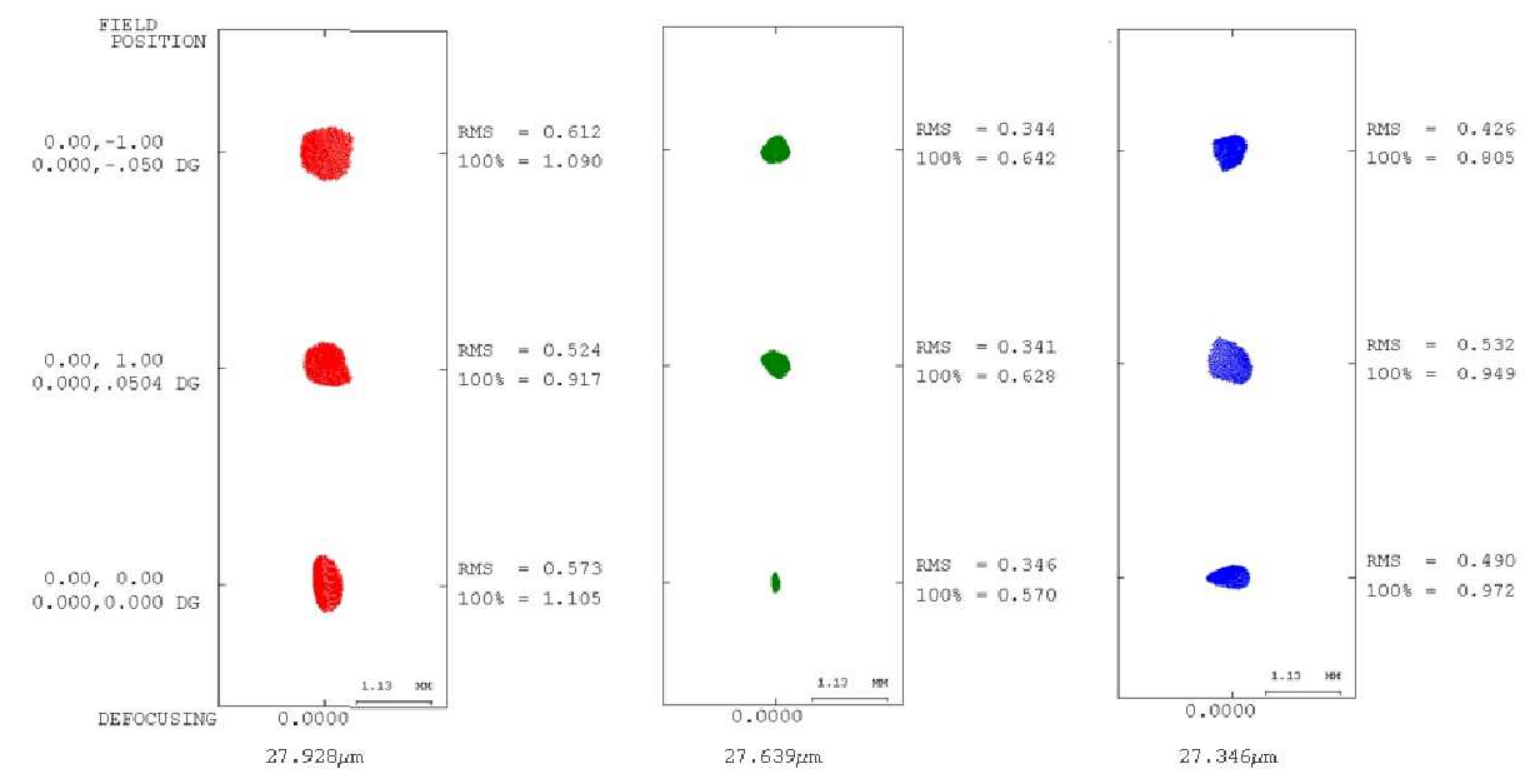

Figure 8. Spot size across the detector (spatial), from $\lambda \min$ to $\lambda \max$ (spectral)

System Resolution is directly related to the incident angle of the grating, with a maximum $\mathrm{R}$ of $\sim 6,000$ at $70^{\circ}$. This is independent of the wavelength range each grating was designed to cover. However, as the wavelength increases, diffraction increases, and the energy passing through the slit and convolved with the detector pixels is further reduced, requiring an opening up of the second field slit and associated binning of pixels at the detector. Figure 9 shows the 2-D effect of wavelength on throughput loss, and the effect of opening the slit width on regaining throughput. Figure 10 shows the numerical values for detector energy as pixels are binned. 


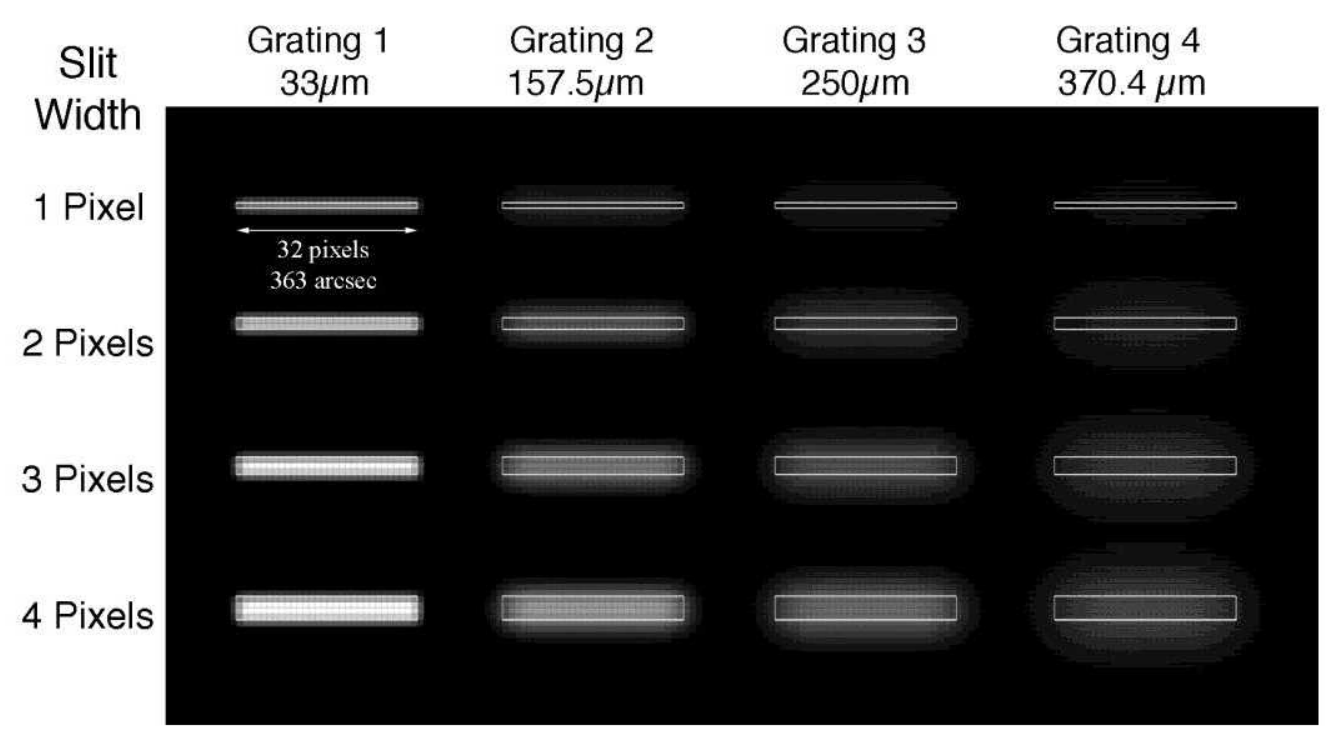

Figure 9. SAFIRE IFOV vs. Slit Width

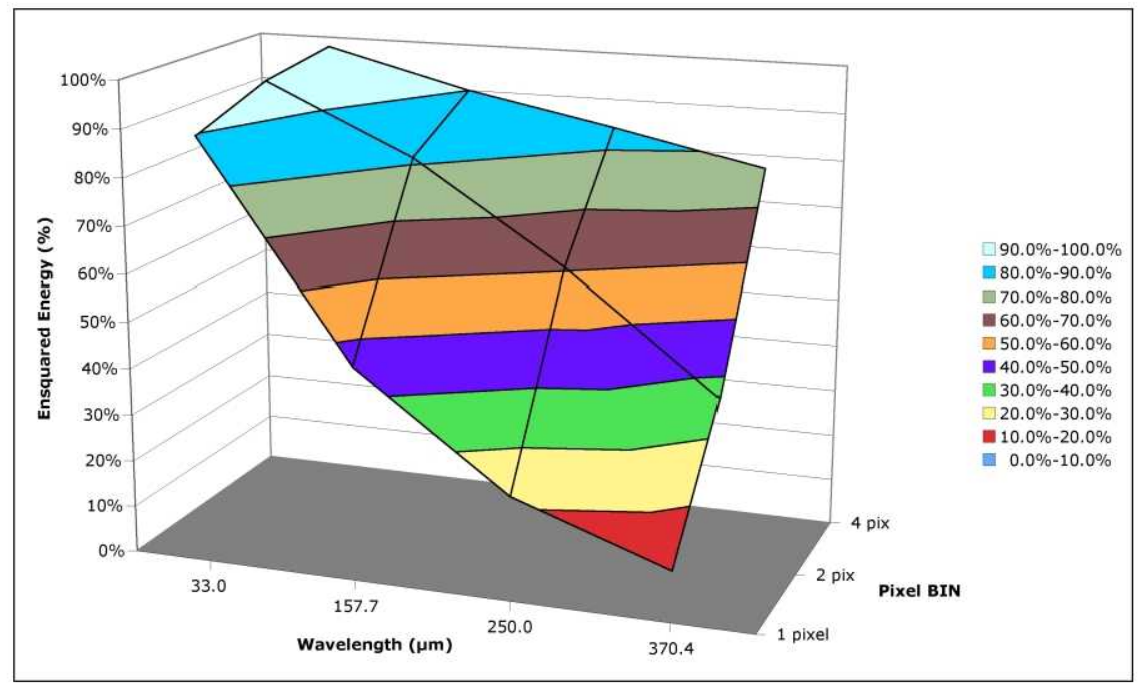

Figure 10. SAFIRE Pixel BINs vs. Detector Energy

The system resolving power is reduced by the pixels binned ( 2 pixels $=$ half $\mathrm{R})$. So to increase throughput at higher wavelengths, resolution would be decreased to $\mathrm{R}=1,000$ to 3,000 . Even with the physical optics limitation, the SAFIRE design for SOPHIA provides a versatile and robust instrument to accomplish the scientific goals of the far-infrared astrophysicist.

\section{REFERENCES}

[1] Shafer, Moseley, Ade, Benford, Bjoraker, Dwek, Neufeld, Pajot, Phillips and Stacey, "Submillimeter and farinfrared experiment (SAFIRE): a PI class instrument for SOFIA," in Airborne Telescope Systems, Proc. SPIE 4014, 98-108 (2000).

[2] Khan, Shafer, Benford, Staguhn, Chanial, Le Floc'h, Babbedge, Farrah, Moseley, Dwek, Clements, Sumner, Ashby, Brand, Brodwin, Eisenhardt, Elston, Gonzalez, McKenzie and Murray, "On the discovery of the first galaxy selected at 350 microns," The American Astronomical Society The Astrophysical Journal, 631:L9-L12 (2005). 